\title{
Effects of an Acute Strength and Conditioning Training Session on Dual Energy X-ray Absorptiometry Results
}

\author{
SEAN T. STANELLE, JASON R. LYTLE, STEVEN E. MARTIN, JOHN S. GREEN, \\ FACSM, and STEPHEN F. CROUSE, FACSM
}

Applied Exercise Science Laboratory; Health and Kinesiology; Texas A\&M; College
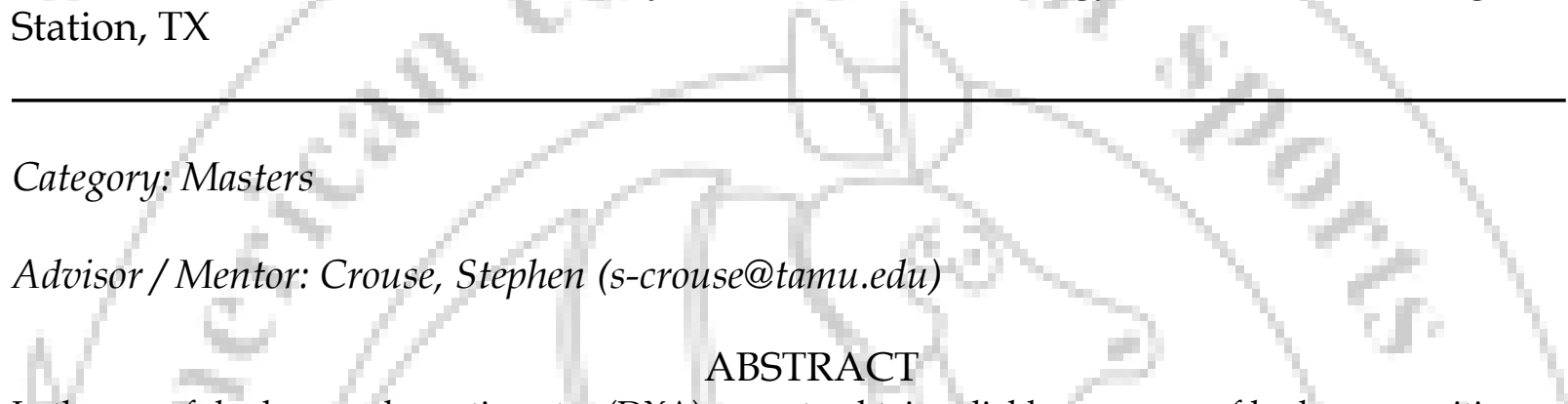

In the use of dual x-ray absorptiometry (DXA) scans to obtain reliable measures of body composition, athletic staff must be aware of acute factors that may alter scan estimates. Although factors such as hydration status and food intake have been shown to alter DXA results (Tinsley, MSSE 2016), it is unknown whether an acute strength and conditioning (S\&C) session will alter DXA scan estimates. PURPOSE: To determine if a strength and conditioning (S\&C) training session, based upon what athletes regularly engage in, will alter body composition estimates (lean mass, fat mass, and bone mineral content) of a DXA scan. METHODS: The S\&C session lasted $\sim 90$ minutes and consisted of upper and lower body resistance exercises and interval running. Twenty-two strength-trained subjects ( 15 men, 7 women, age 24 \pm 2 yrs, height $174.2 \pm 8.5 \mathrm{~cm}$, weight $83.5 \pm 15.0 \mathrm{~kg}$ ) volunteered to participate in the study. A food $\log$ was distributed during the informed consent process, which participants maintained for 24 hours prior to the DXA scans. Each subject completed two standard DXA scans on the same day, before and within 45 minutes of completing the S\&C session. Participants were instructed to consume a normal, free-living breakfast prior to the first scan, and to then avoid all food intake until completing the second scan. Throughout the S\&C session, subjects were encouraged to drink water ad libitum. RESULTS: No significant difference was found on any of the total body measures between pre and post DXA body composition measurements except for total mass, which was found to be lower after the S\&C session (pre to post: $83.8-83.5 \mathrm{~kg}$ ). Compartmental results showed significant differences between pre and post scans in the arms, legs, and trunk. Arm and leg \% fat were found to be lower (pre to post: arm \% fat 20.5-19.9, leg \% fat 23.2-22.6); arm total and lean mass, and leg lean mass were found to be higher (pre to post: arm total mass $10.8-11.0 \mathrm{~kg}$, arm lean mass $8.3-8.5 \mathrm{~kg}$, leg lean mass $21.5-21.8 \mathrm{~kg}$ ); and trunk lean mass was found to be lower (pre to post: $28.7-28.2 \mathrm{~kg}$ ) after the $S \& C$ session. CONCLUSION: Based on the results of the present study, the acute physiological effects of a $S \& C$ session alter body composition measures obtained by DXA scan. Thus, athletic staff should consider the timing of DXA scans in relation to S\&C sessions.

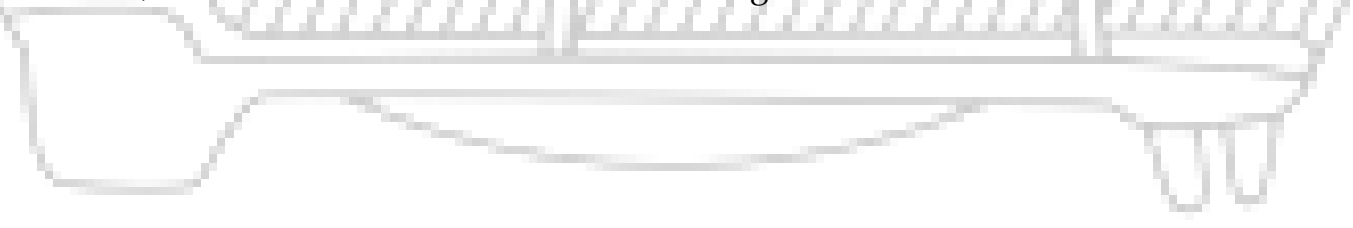

